\title{
NEUTRINOS FROM KALUZA-KLEIN DARK MATTER ANNIHILATIONS IN THE SUN
}

\author{
TOMMY OHLSSON* \\ Department of Theoretical Physics, School of Engineering Sciences, Royal Institute of \\ Technology (KTH) \\ Roslagstullsbacken 21, SE-106 91 Stockholm, Sweden \\ *E-mail: tommy@theophys.kth.se
}

\begin{abstract}
In this talk, we compute fluxes of neutrinos from Kaluza-Klein dark matter annihilations in the Sun based on cross-sections from both five- and six-dimensional models. For our numerical calculations, we use WimpSim and DarkSUSY. In addition, we compare our results with the ones derived earlier in the literature.
\end{abstract}

Keywords: Dark matter theory; Extra dimensions; Neutrino astronomy

\section{Introduction}

Let us shortly motivate the investigation of dark matter (DM) in the Universe. Cosmological and astronomical observations have measured the energy budget of the Universe to be $4 \%$ ordinary baryonic matter, $23 \%$ dark matter, and $73 \%$ dark energy. ${ }^{1}$ Since the DM constitutes approximately a quarter of the total energy budget of the Universe, it is therefore of interest to study its importance. One of the most plausible DM candidates are Weakly Interacting Massive Particles (WIMPs). In particular, neutralinos $(\chi)$ are promising WIMP candidates.

In this talk, we will however study Kaluza-Klein (KK) particles, which are another type of WIMPs. Now, extra-dimensional field theory is non-renormalizable, which means that we have to view such a theory as an effective theory. Therefore, there is a need for a UV completion of the theory. KK particles arise in models with extra dimensions. If so-called KK parity is conserved, then the lightest KK particle (LKP) is stable ( $c f$. , LSP in supersymmetry). If neutral, the LKP can be a good DM candidate. In this part of the proceedings, we abbreviate Kaluza-Klein dark matter by KKDM. In addition, see related talks by Neubert and Volkas at this conference.

What about WIMP capture and annihilation in the Sun as well as neutrino production and detection? WIMPs in the Milky Way halo can scatter in the Sun and be gravitationally bound to it. Eventually, they will scatter again and sink to the core of the Sun. In the core, WIMPs (here: KKDM) will accumulate and can annihilate and produce neutrinos. Only $\nu$ 's can escape the Sun (from WIMP 
annihilations). In the propagation from production in the Sun to detection at the Earth, neutrino oscillations are used. Then, muons are induced by $\nu$ 's in Earth matter. Therefore, fluxes of muons are detected at Earth. Note that we use the DarkSUSY and WimpSim packages to compute muon fluxes at an Earth-based detector.

\section{Extra-Dimensional Models}

In extra-dimensional models, WIMPs are KKDM particles. Especially, in UED models, all SM particles are allowed to propagate in one or more extra dimensions. ${ }^{2}$ In the so-called minimal UED model, i.e., the MUED model, the LKP is the first mode of the U(1) gauge boson. However, we investigate five- and six-dimensional UED models that are based on the SM gauge group and have more general mass spectra than the MUED model. In our analyses, we have made the following approximations: i) all SM particles are assumed to be massless, ii) we ignore EWSB effects, iii) we neglect Yukawa couplings, since they give negligible contributions for the processes of interest (even for the top quark Yukawa coupling), and finally, iv) we ignore self-couplings of the Higgs boson, since none of the studied processes involve this interaction.

\subsection{Five Dimensions}

In five dimensions, spinors are four-component objects. However, there is no chirality operator, which implies that the Dirac representation is irreducible. Thus, this means that the simplest choice of geometry, the circle $S^{1}$, for the fifth dimension does not work. Nevertheless, the orbifold $S^{1} / \mathbb{Z}_{2}$ does the job. For gauge fields in the extra dimensions, there is an additional component $A_{5}$. In four dimensions, the zero mode of such a gauge field appears as a massless scalar. Therefore, we take $A_{5}$ to be odd in $y$, i.e., no zero mode exists.

In general, KK expansions of fields are given by

$$
\begin{aligned}
A^{\text {(even) }}\left(x^{\mu}, y\right) & =\frac{1}{\sqrt{\pi R}}\left[A^{(0)}\left(x^{\mu}\right)+\sqrt{2} \sum_{n=1}^{\infty} \cos \left(\frac{n \pi y}{R}\right) A^{(n)}\left(x^{\mu}\right)\right], \\
A^{\text {(odd })}\left(x^{\mu}, y\right) & =\sqrt{\frac{2}{\pi R}} \sum_{n=1}^{\infty} \sin \left(\frac{n \pi y}{R}\right) A^{(n)}\left(x^{\mu}\right),
\end{aligned}
$$

where the index $n=1$ gives the first KK modes. In addition to the SM parameters, the compactification radius $R$ and the cut-off scale $\Lambda$ are the only free parameters. Here, we ignore effects of KK modes higher than the first-level $(n=1)$ mode. In 
addition, the gauge part of the five-dimensional Lagrangian is

$$
\begin{aligned}
\mathcal{L}_{\text {gauge }} & =-\frac{g}{2} f^{a b c} F_{\mu \nu}^{(0), a} A^{(1), b \mu} A^{(1), c \nu} \\
& -\frac{g}{2} f^{a b c}\left(\partial_{\mu} A_{\nu}^{(1), a}-\partial_{\nu} A_{\mu}^{(1), a}\right)\left(A^{(0), b \mu} A^{(1), c \nu}+A^{(0), c \nu} A^{(1), b \mu}\right) \\
& -\frac{g^{2}}{4}\left[f^{a b c}\left(A_{\mu}^{(0), b} A_{\nu}^{(1), c}+A_{\nu}^{(0), c} A_{\mu}^{(1), b}\right)\right]^{2} .
\end{aligned}
$$

In five dimensions, the possible DM candidates are the first KK modes of neutrinos, the two neutral components of the Higgs doublet, and the $B$ and $W^{3}$ bosons. However, KK neutrinos are ruled out as DM and scalar DM is not interesting in this context, since it has no spin-dependent interactions. Thus, the only interesting DM candidates are $B^{(1)}$ and $W^{3(1)}$.

Some comments on so-called boundary localized terms (BLTs) are in order. In general, orbifold fixed points imply BLTs, which lead to momentum nonconservation in the extra dimensions. However, this means conservation of KKparity. Here, BLTs are included in the Lagrangian and they i) affect the spectrum (at tree level), which means that we can have different LKPs, ii) affect the coupling constants (at tree level), which we have not taken into account, iii) are not determined by the SM parameters, and iv) decrease predictivity of the models.

\subsection{Six Dimensions}

In six dimensions, spinors are eight-component objects, and as in four dimensions, there is a chirality operator. Here, the orbifold is the chiral square $T^{2} / \mathbb{Z}_{4}$.

In general, KK expansion of the fields are given by

$$
A\left(x^{\mu}, x^{4}, x^{5}\right)=\frac{1}{L}\left[\delta_{n, 0} A^{(0,0)}\left(x^{\mu}\right)+\sum_{j \geq 1} \sum_{k \geq 0} f_{n}^{(j, k)}\left(x^{4}, x^{5}\right) A^{(j, k)}\left(x^{\mu}\right)\right],
$$

where

$$
\begin{aligned}
f_{n}^{(j, k)}\left(x^{4}, x^{5}\right) & =\frac{1}{1+\delta_{j, 0}}\left[e^{-\mathrm{i} n \pi / 2} \cos \left(\frac{j x^{4}+k x^{5}}{R}+\frac{n \pi}{2}\right)\right. \\
& \left. \pm \cos \left(\frac{k x^{4}-j x^{5}}{R}+\frac{n \pi}{2}\right)\right]
\end{aligned}
$$

Here the indices $(j, k)=(1,0)$ give the first KK modes. In this case, the gauge part of the five-dimensional Lagrangian is

$$
\begin{aligned}
\mathcal{L}_{\text {gauge }} & =-g f^{a b c} \delta_{0,0,0}^{j_{1}, j_{2}, j_{3}} A_{\mu}^{\left(j_{1}\right), a} A_{\nu}^{\left(j_{2}\right), b} \partial^{\mu} A^{\left(j_{3}\right), c \nu} \\
& +\left(\frac{g}{2} f^{a b c} A_{H}^{(1), a}\left(\partial^{\mu} A_{H}^{(1), b}\right) A_{\mu}^{(0), c}+\text { h.c. }\right) \\
& -\frac{g^{2}}{4} f^{a b c} f^{a d e} \delta_{0,0,0,0}^{j_{1}, j_{2}, j_{3}, j_{4}} A_{\mu}^{\left(j_{1}\right), b} A_{\nu}^{\left(j_{2}\right), c} A^{\left(j_{3}\right), d \mu} A^{\left(j_{4}\right), e \nu} \\
& -\frac{g^{2}}{2} f^{a b c} f^{a d e} A_{H}^{(1), c} A_{H}^{(1), e} A_{\mu}^{(0), b} A^{(0), d \mu}
\end{aligned}
$$


In six dimensions, the possible DM candidates are the same as in five dimensions, and in addition, the first-level adjoint scalars $B_{H}^{(1)}$ and $W_{H}^{3(1)}$ belong to the candidates. However, adjoint scalar DM is not interesting. Thus, in conclusion, the interesting DM candidates are the same as in five dimensions.

\section{Capture Rates, Branching Ratios, and IceCube}

In this section, we discuss capture rates and compare the result from an approximative formula given by

$$
C_{\text {approx }} \simeq 3.35 \cdot 10^{18} \mathrm{~s}^{-1}\left(\frac{\rho}{0.3 \mathrm{GeV} / \mathrm{cm}^{3}}\right)\left(\frac{270 \mathrm{~km} / \mathrm{s}}{\bar{v}}\right)^{3}\left(\frac{\sigma_{\mathrm{WIMP}, \mathrm{p}}^{\mathrm{SD}}}{10^{-6} \mathrm{pb}}\right)\left(\frac{1 \mathrm{TeV}}{m_{\mathrm{WIMP}}}\right)^{2}
$$

and the result from DarkSUSY. In Fig. 1, the ratio of the two different capture rates is shown as a function of the WIMP mass $m_{\text {WIMP }}$. We observe that there is a difference between the two capture rates that is about $25 \%$.

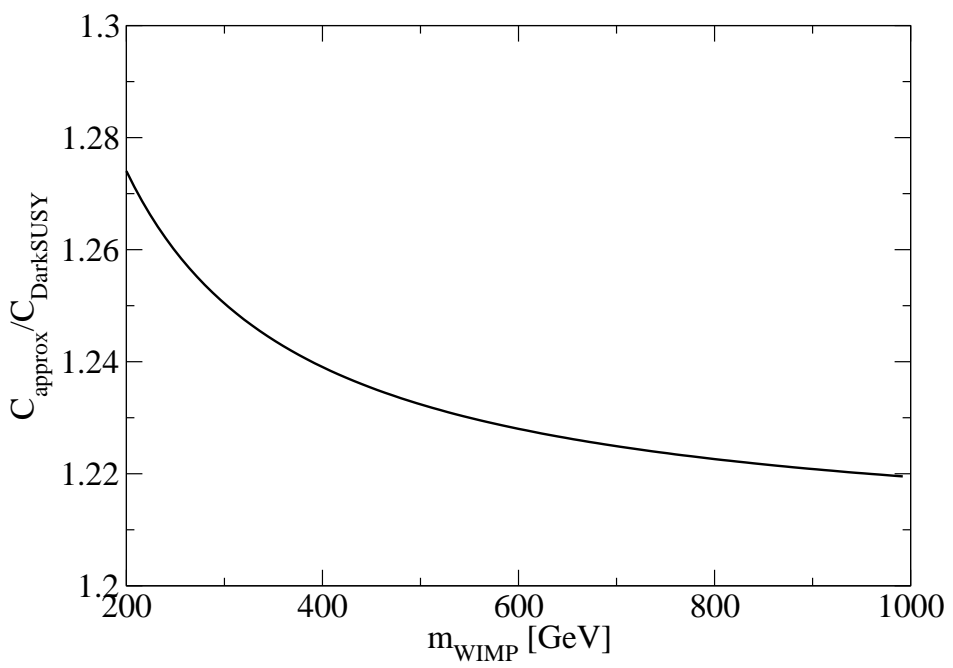

Fig. 1. The ratio of the capture rates as a function of the WIMP mass $m_{\text {WIMP }}$ [as obtained using Eq. (7) and DarkSUSY]. This figure has been adopted from Ref. ${ }^{3}$

In Table 1, we present branching ratios for pair annihilations of KKDM into different final states. The values of the branching ratios have been computed for two different values (1.0 and 1.3) of the relative mass splitting between the LKP and the KK quarks, which is defined as

$$
r_{q} \equiv \frac{m_{q^{(1)}}-m_{\mathrm{LKP}}}{m_{\mathrm{LKP}}} .
$$

We observe that the branching ratios into a pair of Higgs bosons are small and they contribute only with a tiny fraction to the muon-antimuon fluxes. In addition, we 
have included in the table the corresponding branching ratios, which were found in Ref. ${ }^{4}$ Especially, we note that the values of the branching ratios into $W^{+} W^{-}$are different in our calculations compared with the values given in Ref. ${ }^{4}$

Table 1. Branching ratios for pair annihilations of KKDM.

\begin{tabular}{|l||l|l|l|l|l|l|}
\hline \multicolumn{1}{|l||}{ Final state } & \multicolumn{2}{c|}{$B^{(1)}$} & \multicolumn{2}{c|}{$W^{3(1)}$} & $B^{(1)}$ & $W^{3(1)}$ \\
\hline$r_{q}$ & 1.0 & 1.3 & 1.0 & 1.3 & \multicolumn{2}{|c|}{ Ref. $^{4}$} \\
\hline \hline $\bar{u} u$ & 0.125 & 0.084 & 0.017 & 0.010 & 0.04 & 0.043 \\
$\bar{d} d$ & 0.008 & 0.006 & 0.017 & 0.010 & 0.04 & 0.043 \\
$\bar{\nu} \nu$ & 0.011 & 0.013 & 0.005 & 0.005 & 0.013 & 0.013 \\
$l^{+} l^{-}$ & 0.183 & 0.223 & 0.005 & 0.005 & 0.20 & 0.01 \\
$h h$ & 0.004 & 0.005 & 0.002 & 0.002 & $\times$ & $\times$ \\
$Z Z$ & 0.004 & 0.005 & 0.002 & 0.002 & $\times$ & $\times$ \\
$W^{+} W^{-}$ & 0.010 & 0.012 & 0.866 & 0.908 & 0 & 0.65 \\
\hline
\end{tabular}

In Fig. 2, the bounds on the muon-antimuon flux at IceCube from LKP annihilations the Sun are presented. We will include the bounds found by the IceCube

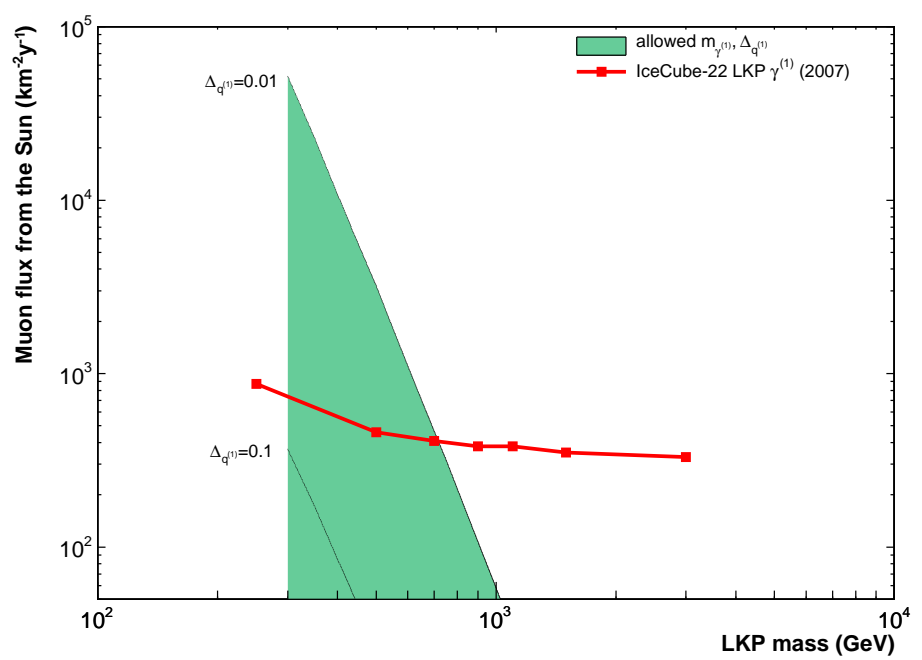

Fig. 2. Limits on the muon flux from LKP annihilations in the Sun including systematic errors (squares). This figure has been adopted from Ref. ${ }^{5}$

collaboration in our results.

\section{Results}

In this section, we present the results of our computations of the muon-antimuon flux in a detector at Earth from the Sun. In Fig. 3, the left plot shows the results 
for $B^{(1)}$ being the LKP, whereas the right plot shows the results for $W^{3(1)}$ being the LKP. ${ }^{3}$ For both plots, a muon energy threshold $E_{\mu}^{\text {th }}=1 \mathrm{GeV}$ has been used. Note that the thicker curve segments show the LKP mass range that reproduces correct relic abundance.
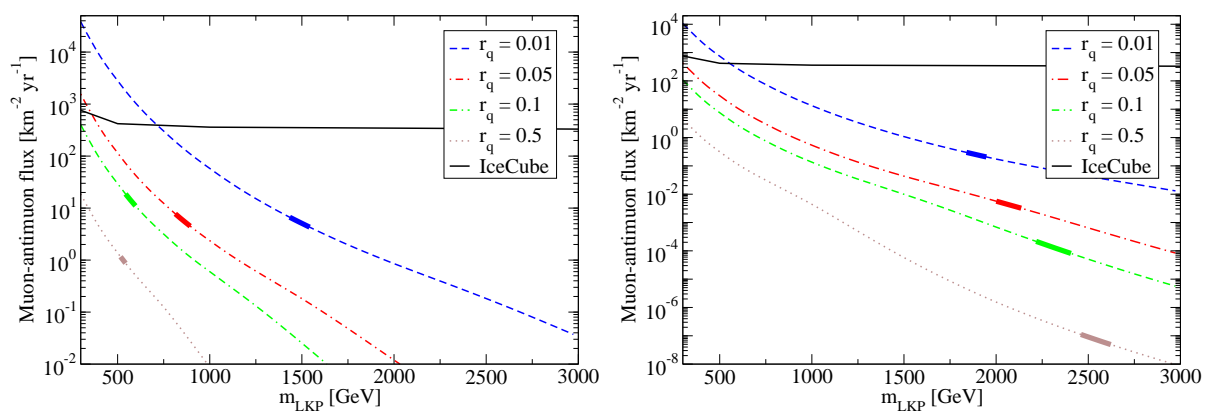

Fig. 3. Left: The muon-antimuon flux in a detector at Earth as a function of the WIMP mass $m_{\text {WIMP }}$ for $B^{(1)}$ being the LKP. Right: The muon-antimuon flux in a detector at Earth as a function of the WIMP mass $m_{\text {WIMP }}$ for $W^{3(1)}$ being the LKP. These figures have been adopted from Ref. ${ }^{3}$

Finally, we comment shortly on earlier results obtained in the literature. Neutrinos from KKDM annihilations in the Sun have previously been studied by: D. Hooper and G.D. Kribs ${ }^{6}$ and T. Flacke, A. Menon, D. Hooper, and K. Freese. ${ }^{4}$ Our study ${ }^{3}$ is a more careful treatment, it includes a six-dimensional model, it gives different branching ratios for $W^{3(1)}$, and it results in a difference of $20 \%-$ $30 \%$. In addition, the IceCube collaboration (see Ref..$^{5}$ ) has computed fluxes for the five-dimensional MUED model, which are similar to our results.

\section{Summary and Conclusions}

We have investigated KKDM in two extra-dimensional models - one fivedimensional model and one six-dimensional model. In both models, $B^{(1)}$ and $W^{3(1)}$ as LKPs are the interesting DM candidates. We have calculated the flux of neutrinoinduced muons and antimuons in an Earth-based neutrino telescope (e.g. IceCube). The fluxes for the five- and six-dimensional models are equal. Therefore, it is not possible to distinguish them. The flux of neutrinos is somewhat larger for $B^{(1)}$ than for $W^{3(1)}$. If $B^{(1)}$ is the LKP, IceCube can put constraints on the parameter space. However, not if $W^{3(1)}$ is the LKP.

\section{Acknowledgments}

I would like to thank my collaborators Mattias Blennow and Henrik Melbéus for useful collaboration that led to the publications upon which this talk is based. In addition, I would like to thank the organizers of 5th International Conference 
on Beyond the Standard Models of Particle Physics, Cosmology, and Astrophysics (Beyond 2010) for the invitation.

This work was supported by the Royal Swedish Academy of Sciences (KVA) and the Swedish Research Council (Vetenskapsrådet), contract no. 621-2008-4210.

\section{References}

1. E. Komatsu et al., Astrophys. J. Suppl. 180, 330 (2009).

2. T. Appelquist, H.-C. Cheng and B. A. Dobrescu, Phys. Rev. D64, 035002 (2001).

3. M. Blennow, H. Melbéus and T. Ohlsson, JCAP 1001, 018 (2010).

4. T. Flacke, A. Menon, D. Hooper and K. Freese, Kaluza-Klein dark matter and neutrinos from annihilation in the Sun, arXiv:0908.0899 [hep-ph], (2009).

5. R. Abbasi et al., Limits on a muon flux from Kaluza-Klein dark matter annihilations in the Sun from the IceCube 22-string detector, arXiv:0910.4460 [astro-ph.CO], (2009).

6. D. Hooper and G. D. Kribs, Phys. Rev. D67, 055003 (2003). 